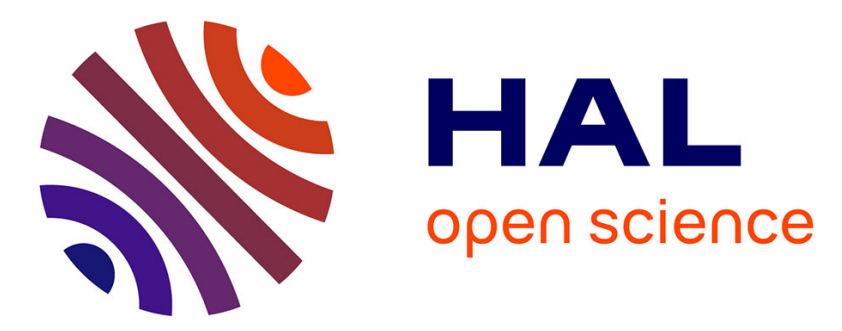

\title{
Bond graph modeling and analysis of intermediary cooling system of a nuclear power plants
}

Toufik Bentaleb, Minh Tu Pham, Damien Eberard, Wilfrid Marquis-Favre

\section{To cite this version:}

Toufik Bentaleb, Minh Tu Pham, Damien Eberard, Wilfrid Marquis-Favre. Bond graph modeling and analysis of intermediary cooling system of a nuclear power plants. 2018 IEEE ICIT, Feb 2018, Lyon, France. 10.1109/icit.2018.8352158 . hal-01863651

\section{HAL Id: hal-01863651 https://hal.science/hal-01863651}

Submitted on 12 Apr 2019

HAL is a multi-disciplinary open access archive for the deposit and dissemination of scientific research documents, whether they are published or not. The documents may come from teaching and research institutions in France or abroad, or from public or private research centers.
L'archive ouverte pluridisciplinaire HAL, est destinée au dépôt et à la diffusion de documents scientifiques de niveau recherche, publiés ou non, émanant des établissements d'enseignement et de recherche français ou étrangers, des laboratoires publics ou privés. 


\section{Bond graph modeling and analysis of intermediary cooling system of a nuclear power plants}

\author{
Toufik Bentaleb \\ Chair of Applied Dynamics \\ University of Erlangen-Nuremberg \\ Immerwahrstrasse 1, 91058 Erlangen, Germany \\ toufik.bentaleb@fau.de
}

\author{
Minh Tu Pham, Damien Eberard, and Wilfrid Marquis-Favre \\ INSA, CNRS, AMPERE \\ University of Lyon \\ F-69100, VILLEURBANNE, France \\ name.surname@insa-lyon.fr
}

\begin{abstract}
This paper presents a bond graph (BG) model and a physical structural analysis of an intermediary cooling system of a power plant. The main goal is to extend the techniques already in use for the physical structural analysis of mechatronic systems to perform static analysis and diagnosis on physical models in order to state whether they are well-posed and suitable for each step of the system's lifecycle (from its design to its operation). If a problem for modeling, for simulating or for solving the given engineering question is detected, the analysis shall localize the problem in the model and give the physical interpretation of the error. The objective is not to be able to diagnose all possible physical errors, but to add another type of analysis in order to improve error detection before compiling and running a model.

Index Terms-bond graph model; intermediary cooling system; nuclear power plants
\end{abstract}

\section{INTRODUCTION}

Physical structural analysis should be viewed as complementary to numerical simulation but a valuable model-based approach for giving information about how far a model is suitable to answer to an engineering problem (model inversion, input reconstruction, control input synthesis, state estimation, parameter identification,...) Interestingly enough, by its nature physical structural analysis is undertaken before simulation and even before the model source compiling. It is the step just after fixing the assumptions and both the energy architecture and the phenomena behavior laws of the system model. The first great interest of physical structural analysis is that it relates the model capability features to answer to an engineering problem with the physical system information. A second interest is that when the properties or the criteria are not satisfied at this stage, it is not needed to pursue an useless numerical analysis. A third interest is that the physical structural analysis gives guidelines to where and how to reformulate the model for it to suit the engineering problem when some criteria are not satisfied.

One of the goals of this work is to extend the techniques already in use for the physical structural analysis of mechatronic systems to perform static analysis and diagnosis on physical models in order to state whether they are well-posed and suitable for each step of the system's lifecycle (from its design to its operation). If a problem for modeling, for simulating or for solving the given engineering question is detected, the analysis shall localize the problem in the model and give the physical interpretation of the error. The objective is not to be able to diagnose all possible physical errors, but to add another type of analysis in order to improve error detection before compiling and running a model. The analysis is based on bond graph representation which is built using the graphical editor MS1 [1]-[3]. The physical structural analysis is based on the system's energy structure [4].

This work is an extended of work done by our group that presented in [5]. In our previous work, we have presented the multi-port pseudo BG model of a plate heat exchanger system which is one component of the intermediary cooling system. The comparison of the simulation results of bond graph model with the Modelica [6] model indicates that the model predicts the dynamic behavior of the heat exchanger well. The main objective in this paper is to develop and analyze the bond graph of the rest of the components without comparing them to the Modelica models.

The description of the physical model of the intermediary cooling system is given in Section [I] The Section [II presents the component models in bond graph, then the physical structural analysis of each component is undertaken in Section IV Finally, Section $\mathrm{V}$ concludes the paper with giving the guidelines for future work.

\section{Physical MOdel OF THE INTERMEdiaRY COOLING SYSTEM}

An intermediary cooling system is part of the intermediate cooling-water of some power plants. The intermediary cooling system is one important subsystem composed of 4 sub-systems (Figure 11): the cooling system, with two heat exchangers and two regulation valves; the water circulation system, made of 2 pumps (in parallel); the water feeding system with a feeding tank; the auxiliary equipment standing for the load of the system with a source of heat and a user valve. Furthermore the following assumptions are considered: the heat intake is given; the valves opening are given (users' valve); the initial fluid height in the feeding tank is known and never goes down under the outlet orifice; the orifice diameters in the feeding tank are not taken into account; all the pressure and temperature boundary conditions are given; there is no water flow inversion in any part of the circuit (so no need of check valves); there is no water flow threshold effect; there is no change of water 


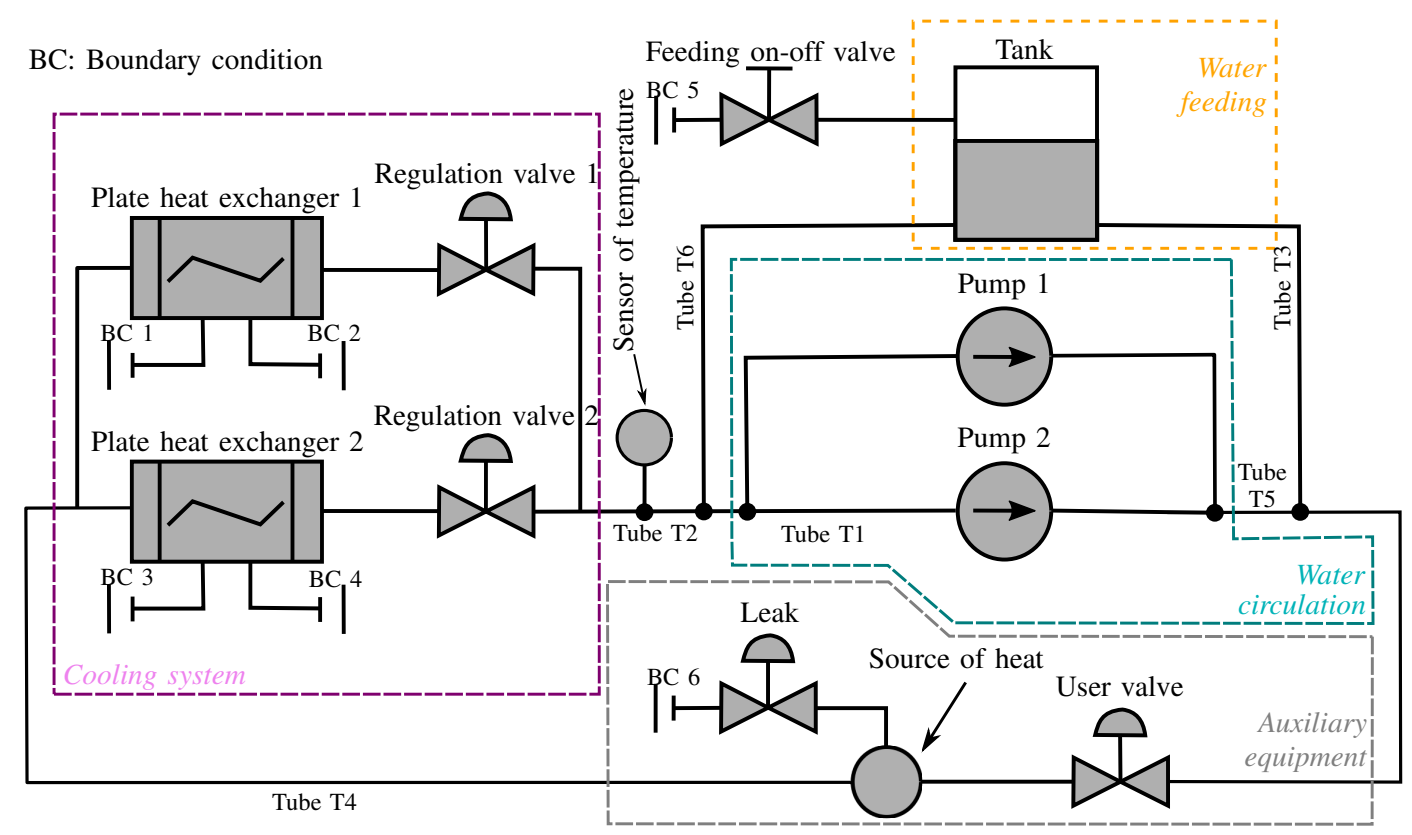

Figure 1. Schematic description of the intermediary cooling system

phase which stays liquid; there is no leak in the auxiliary equipment.

\section{BOND-GRAPH MODEL OF THE INTERMEDIARY COOLING SYSTEM COMPONENTS}

The bond graph model of this complex dynamic behavior and nonlinear system in thermofluids engineering allows to non-expert to get a better understanding of the model and to analyse the properties of this physical system [2], [7]. In this approach, the specific enthalpy and some other properties of water are calculated from tables depending on pressure and temperature.

\section{A. Feeding tank}

The feeding tank prevents the lack of water in the cooling circuit. The bond graph representation Figure 2 makes explicit that the feeding tank component exhibits a two-port energy storage ( $\mathbb{C}$ element) and three multiport energy dissipations $(\mathbb{R}$ elements) connected respectively to the feeding valve, tube 6 and tube 3 components (see Tube T 6 and Tube T 3 in the Figure [1). The energy storage is associated to the state of the water inside the tank. The corresponding energy variables are the mass $m$ and the specific enthalpy $h$ of water. The incompressibility assumption and the state equations for the water volume give the following relationships [8]:

$$
\left\{\begin{array}{l}
\rho \cdot A \cdot \frac{\mathrm{d} z}{\mathrm{~d} t}=\dot{m} \\
\rho \cdot A \cdot z \cdot \frac{\mathrm{d} h}{\mathrm{~d} t}+h \cdot \dot{m}=\dot{H}
\end{array}\right.
$$

where $A$ is the tank cross sectional area, $z$ is the water height, $h$ is the water specific enthalpy, $\dot{m}$ is the change in mass flow rate, while $\rho, H$ are the water density and enthalpy in the tank, respectively.

The energy storage behavior laws give the relationships between the energy variables and the conjugated power variables:

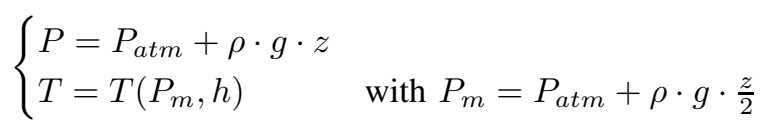

The 0 -junctions are associated to the conservation laws of the water volume that ideally furnishes $\dot{m}$ and $\dot{H}$ respectively to the energy storage phenomenon if the latter is in integral causality:

$$
\left\{\begin{array}{l}
\dot{m}=\sum_{i=1}^{2} q_{\text {in }, i}-q_{\text {out }} \\
\dot{H}=\sum_{i=1}^{2} q_{\text {in }, i} \cdot h_{\text {in }, i}-q_{\text {out }} \cdot h_{\text {out }}
\end{array}\right.
$$

where $q_{j}$ the mass flow rate with $j$ the subscript indicating the orifice "from feeding valve" (in, 1), "from Tube $\mathrm{T} 6$ " $($ in, 2$)$ or "to Tube T3" (out). In (3) the mass conservation is represented in left-hand side 0 -junction, while the energy conservation in the right-hand.

In integral causality the energy storage behavior laws furnish the pressure $P$ and the temperature $T$ to the dissipation phenomena through the 0 -junctions. The dissipation phenomena are associated with the singular pressure drops through the inlet and outlet orifices. Each of their corresponding behavior laws is associated to the following equation:

$$
k \cdot \frac{q_{i} \cdot\left|q_{i}\right|}{2 \rho}=\max \left(P-\rho \cdot g \cdot z_{i}, P_{a t m}\right)-P_{i}
$$


where $k$ is the inlet/outlet pressure loss coefficient, $P_{a t m}$ the atmospheric pressure, $g$ the gravity constant, $P_{i}$ the fluid pressure just beyond the orifice, and $z_{i}$ the orifice altitude with $i$ the subscript indicating the orifice "from feeding valve" (in, 1), "from Tube T6" (in,2) or "to Tube T3" (out).

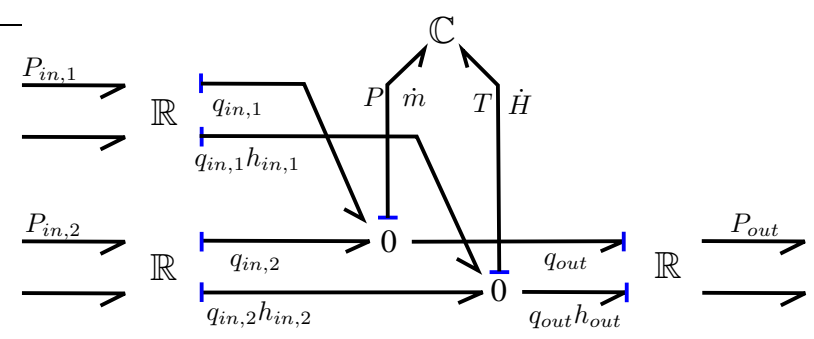

Figure 2. Partial causal bond graph representation of the feeding tank

\section{B. Centrifugal pump}

The pumps ensure the water circulation in the intermediary cooling system circuit. The model presented here is a dynamic pump model, that integrates the rotating mass equation. The bond graph representation of the pump is given in Figure 3 The energy storage phenomenon is reduced to a one-port Celement since the pump volume $V$ is constant so as it is supposed for the water density $\rho$ in this component. Consequently it can be established the relationship between the time derivatives of the specific enthalpy $h$ and the enthalpy $H$ :

$$
\rho \cdot V \cdot \frac{\mathrm{d} h}{\mathrm{~d} t}=\dot{H}
$$

In return the energy storage phenomenon furnishes the temperature $T$ that can be obtained by the water property tables $T(h)$ at the given density $\rho$ [9].

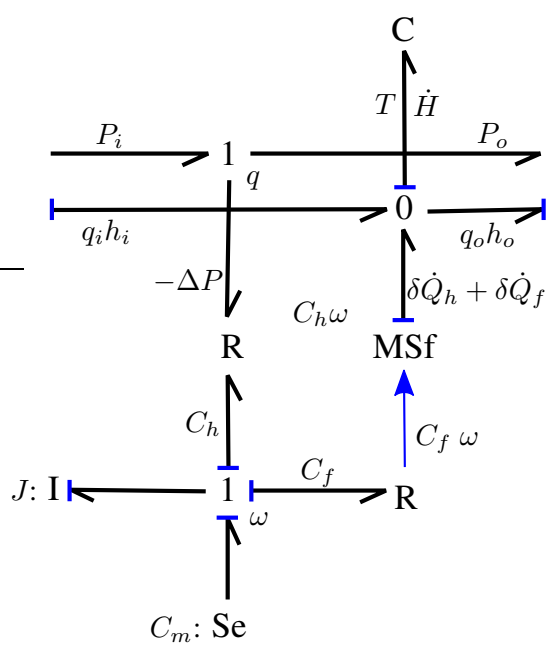

Figure 3. Partial causal bond graph representation of a pump

The unique 0 -junction corresponds to the energy conservation law expressed by:

$$
\dot{H}=q_{i} \cdot h_{i}-q_{o} \cdot h_{o}+\delta \dot{Q}_{h}+\delta \dot{Q}_{f}
$$

where $\delta \dot{Q}_{h}=C_{h} \cdot \omega$ is the heat flow rate due the hydraulic resistance and $\delta \dot{Q}_{f}=C_{f} \cdot \omega$ the heat flow rate due to the mechanical friction on the pump shaft. These terms are furnished by a two- and a one-port dissipation phenomenon respectively (R-elements), which occur in the rotational dynamics of the pump.

The angular velocity $\omega$ is governed by the fundamental law of dynamics for rotational mechanics:

$$
J \cdot \frac{\mathrm{d} \omega}{\mathrm{d} t}=C_{m}-C_{h}-C_{f}
$$

where $J$ is the total rotor inertia, $C_{m}$ the electric drive torque, $C_{h}=C_{h}(\omega, q)$ the resistant hydraulic torque applied by the fluid on the shaft with $q$ the water mass flow rate through the pump, and $C_{f}=C_{f}(\omega)$ the mechanical friction torque on the shaft. This rotational dynamics is represented by a conservation law in (7) associated to a 1 -junction that collects the terms from the energy storage (I-element), the energy source (Se-element) and the dissipation phenomena (Relements).

Finally the pressure drop $\Delta P$ is related to the inlet et outlet pump pressures through the 1 -junction to which is associated the mass flow rate $q$. The dissipation phenomenon gives the last relationship between this pressure drop and both the pump shaft angular velocity $\omega$ and the mass flow rate (by the intermediary of the pump head $h_{n}$ ). The pump head $h_{n}$ is given by:

$$
h_{n}=a_{1} \cdot \frac{1}{\rho^{2}} \cdot q \cdot|q|+a_{2} \cdot \frac{1}{\rho} \cdot q \cdot R+a_{3} \cdot R \cdot|R|
$$

where as above $R=\frac{30 \omega}{\pi \cdot N_{n o m}}$ with $N_{\text {nom }}$ the nominal angular velocity of the pump in rpm, and $a_{i}(i=1,2,3)$ three characteristic constant coefficients.

\section{Valves}

Three types of valves are considered in the intermediary cooling system model: regulation valves at the heat exchanger outlets, the on/off valve for the feeding tank and the users' valve for the auxiliary equipment. All are modeled by singular pressure drops with the same form of dissipation behavior law but with different opening rules. The regulation valves have variable opening depending on the controller law (not modeled here). The users' valve is supposed open at a fixed and maximal given value. The feeding valve is either open or closed. The behavior laws attached to these dissipation phenomena is written into the following forms depending on the variables imposed by the environmental components at the external ports of this phenomena:

$$
\left\{\begin{array}{l}
q=u(t) \cdot C_{v}^{\max } \cdot \frac{\rho}{\sqrt{1.733 \cdot 10^{12}}} \cdot \sqrt{|\Delta P|} \cdot \operatorname{sgn} \Delta P \\
q=C_{v}^{\max } \cdot \frac{\rho}{\sqrt{1.733 \cdot 10^{12}}} \cdot \sqrt{|\Delta P|} \cdot \operatorname{sgn} \Delta P \\
\begin{cases}q=\sqrt{\frac{2 \rho}{k} \cdot|\Delta P|} \cdot \operatorname{sgn} \Delta P & \text { valve on } \\
q=q_{\text {min }} & \text { valve off }\end{cases}
\end{array}\right.
$$

The first equation in (9) is for the regulation valves, the second equation is for the users' valves, and the last eqaution is for the feeding on/off valve. 
The bond graph representation is similar to the one used for dissipation effects at the boundary between two control volume in the heat exchanger or through orifices of the feeding tank since it corresponds to singular pressure drops (Figure 4). It also exhibits a modulation that is the opening law $u(t)$ for the regulation valves, not activated for the users' valve and an on/off signal for the feeding tank.

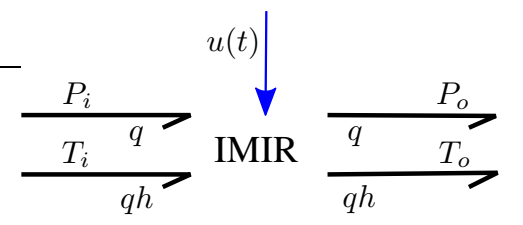

Figure 4. Bond graph representation for the dissipation effects through a valve

\section{Lumped straight pipes (circular duct)}

Every phenomenon continuously distributed all along the pipe is considered localized at one point for approximation. In this model no change of mass flow rate neither the specific enthalpy flow rate is supposed between the inlet and outlet of the pipe. This states:

$$
\left\{\begin{array}{l}
q_{i}=q_{o} \\
h_{i}=h_{o}
\end{array}\right.
$$

where the subscripts $i$ and $o$ stand for inlet and outlet respectively.

The application of the momentum conservation law on the pipe gives the total pipe pressure drop $\Delta P=P_{i}-P_{o}$ :

$\Delta P= \begin{cases}\Delta P_{f}+\rho \cdot g \cdot \Delta z+\frac{L}{A} \cdot \frac{\mathrm{d} q}{\mathrm{~d} t} & \text { with inertia effect } \\ \Delta P_{f}+\rho \cdot g \cdot \Delta z & \text { without inertia effect }\end{cases}$

where $\rho$ is the water density supposed here constant, $g$ the gravity constant, $\Delta z$ the inlet/outlet altitude change, $L$ the pipe length, and $A$ the duct cross-sectional area.

$\Delta P_{f}$ corresponds to the pressure loss due to friction in the pipe. It is expressed by:

$$
\Delta P_{f}=\frac{k}{2 \cdot \rho} \cdot q^{2} \operatorname{sgn} q
$$

where $k$ is the regular pressure drop coefficient.

The bond graph representation of a pipe model is presented in Figure 5. It shows an energy storage (I-element) for the inertia effects along the pipe. If they are not taken into account, this element simply vanishes. The pressure drop due to the gravity effect is represented by an effort source and the one due to friction by an R-element.

The behavior law associated to the energy storage is linear and states that the fluid momentum is proportional to the

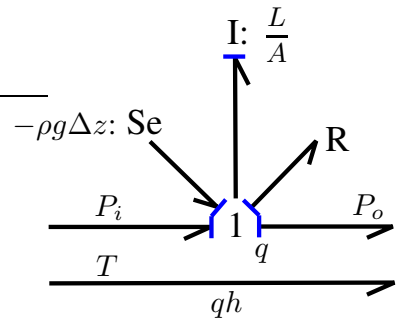

Figure 5. Partial causal bond graph representation of pipe models

mass flow rate where $\frac{L}{A}$ is the coefficient. The behavior law associated to the dissipation effects for friction is given by (12). The bond graph representation proposed for this model consider continuity of mass flow rate, specific enthalpy flow rate and also temperature. If one wishes a temperature gradient to be modeled along the pipe, second port on the dissipation phenomenon can be added and connected to a 1-junction inserted on the thermal domain. In that case this requires the use of water property tables, in particular, to define the water specific enthalpy.

\section{E. Circuit junctions}

The last component to inspect in the intermediary cooling system model is a junction of several pipes at some node of the circuit.

According to the analogy above the bond graph model of a volume for a circuit junction is the same as the Figure 5 in [5] with the corresponding equations but without the bond associated to the heat exchange and as many pairs of thermofluid bonds as the number of merging pipes. If one wishes to idealize the circuit junction neglecting the volume storage effect one simply takes off the C-element leaving only bonds and 0 -junctions.

\section{Structural Physical analysis of INTERmediary COOLING SYSTEM}

\section{A. Feeding tank}

Like for the heat exchangers the characteristics of the storage phenomenon says that there are two state variables both of generalized displacement type. In integral causality they are determined by integrating their time derivatives which are given principally by the conservation laws. In return the behavior laws of the energy storage enable to calculate the conjugated variables. The physics of this component [8] says that mass $m$ and enthalpy $H$ of the water in the feeding tank can be taken as state variables and that the corresponding conjugated variables are respectively the pressure $P$ and the temperature $T$.

Concerning the energy dissipation phenomena, they furnish the different mass flow rates $q_{i}$ and specific enthalpy flow rates $q_{i} h_{i}$ to the conservation laws. In return, they receive the pressure and the temperature in the feeding tank. Depending on the components connected at their "external" ports and the information received through these ports, the dissipation 
phenomena will either determine the temperature or the specific enthalpy flow rate for each thermal port (lower bonds), and either the pressure or the mass flow rate for each fluid mechanics port (upper bonds). This physical structural analysis is clearly displayed by the Figure 2 partial causal bond graph. The causal strokes indicates which variables (effort- or flow-like) are furnished by which phenomena. The absence of strokes at the external ports shows the previously mentioned indetermination at this local analysis level. One important consequence will be the type of water property tables that will be required for the dissipation behavior laws.

\section{B. Centrifugal pump}

The preferred integral causality for the energy storage phenomenon leads to the partial causal assignment of the pump bond graph (Figure 3). This shows that the time derivative of the water enthalpy is furnished to the energy storage phenomenon by the energy conservation law. The energy storage phenomenon returns back the temperature that is transmitted to the pump inlet and outlet.

Concerning the fluid domain, in particular involving the dissipation phenomena, different variable assignments may happen depending on the environmental components connected at the corresponding ports. If the mass flow rate is determined by the connected component at one of the pump fluid ports (either inlet or outlet), one pressure by the one at the other pump fluid port, then the pressure drop is calculated by the dissipation behavior phenomenon which determines the pressure at the first pump fluid port. A second possibility is that both pressures at the pump fluid external ports are imposed by the corresponding connected components. These pressures determine the pressure drop that in turn is used by the dissipation behavior law to furnish the mass flow rate to the external components. This organization of the equations is conditioned by the invertibility of the dissipation behavior law. The expression of the pump head in (8) is probably the main limit to this second variable assignment. This will be subject to discussion in the global phase of physical structural analysis with the connection of the different components.

\section{Valves}

The models of valves presented do not exhibit any storage phenomenon. They have static behaviors. This confers flexibility for assigning the different variables depending on the causality of environmental components that they may impose. Nevertheless it is conditioned by the possibility of inverting the dissipation behavior law i.e. the possibility of equally using (9). Another consequence is once again the water property tables required. For instance the second form of behavior laws in (9) requires the specific enthalpy $h=h\left(P_{m}, T_{m}\right)$ where averaged pressure $P_{m}=\frac{P_{i}+P_{o}}{2}$ and temperature $T_{m}=\frac{T_{i}+T_{o}}{2}$ can be taken. The final choice is done at the global level of the physical structural analysis.

\section{Lumped straight pipes (circular duct)}

The key issue of the physical structure analysis at this component level is the assumption about the inertia effects. If they are considered and that integral causality is preferred, then the energy storage receives the balance of effort-like variables (the pressures at the pipe inlet and outlet, the pressure loss due to friction and the one due to gravity effect to compute the mass flow rate imposed to the pressure drops behavior laws and to the pipe external ports. On the contrary if no inertia effect is supposed then one external connected component has to furnish one pressure and the other one the mass flow rate. The final decision is to be made at the global level of the physical structure analysis.

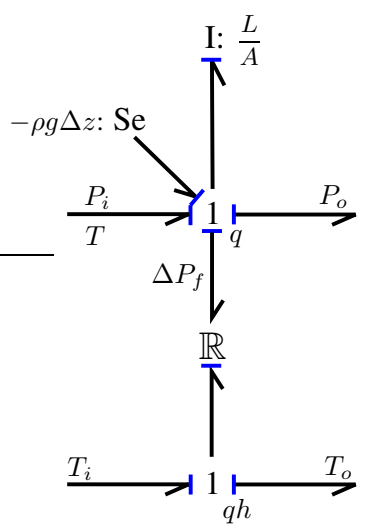

Figure 6. Causal bond graph representation of pipe models

Furthermore the thermal domain representation (lower bond of Figure 5 bond graph) states the continuity of both specific enthalpy flow rate and temperature. If the former is imposed at one external port the temperature cannot be also imposed at the same port. Now if a temperature gradient along the pipe is supposed still with the continuity of specific enthalpy flow rate this may enable to impose both temperatures from the external connected component (Figure 6). In that case specific enthalpy can be obtained from water property tables in termes of an averaged temperature and the water density: $h=h\left(\frac{T_{i}+T_{o}}{2}, \rho\right)$.

\section{E. Circuit junctions}

Due to the analogy the physical structural analysis is similar. The preferred integral causality for the energy storage imposes the effort variables to the environmental connected components: the pressures for the fluid domain and the temperatures for the thermal domain. Figure 8 shows the causal bond graph for a junction with energy storage phenomena in preferred integral causality and three connecting pipes one inlet and two outlets. This feature will be advantageously used at the global level when connecting these junction models to the pipe ones.

The flexibility of neglecting the energy storage phenomena shall also enable to match particular causality requirements 


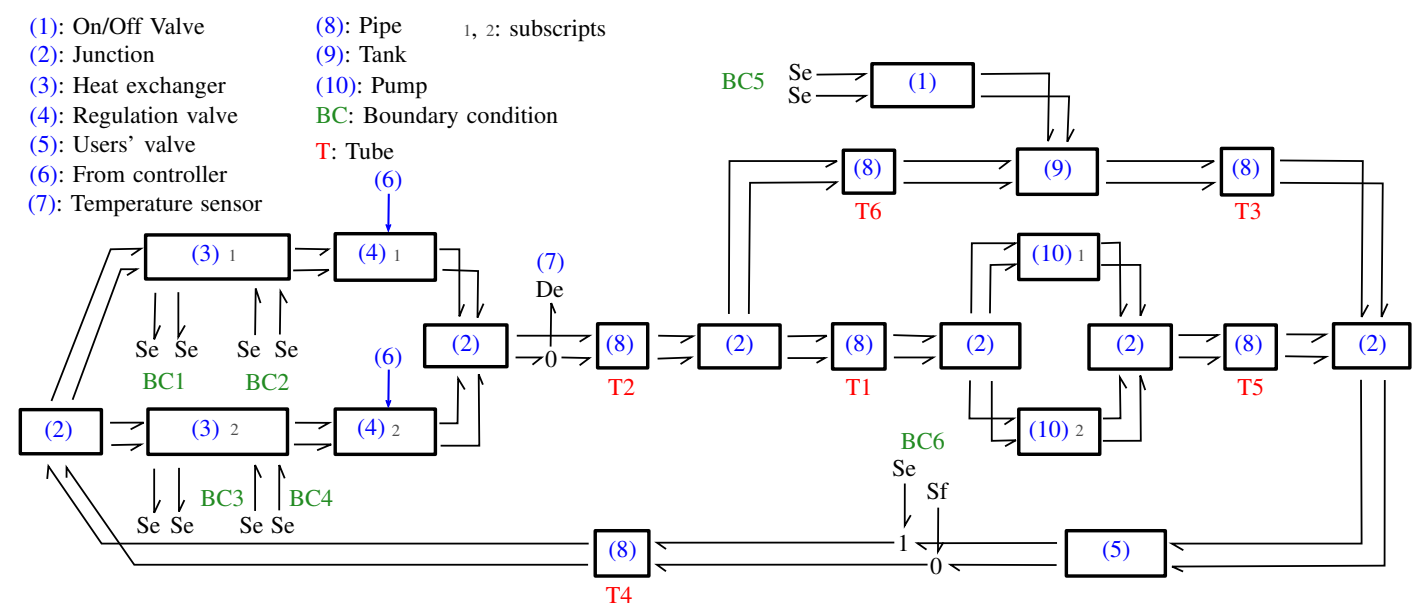

Figure 7. Word bond graph of the intermediary cooling system

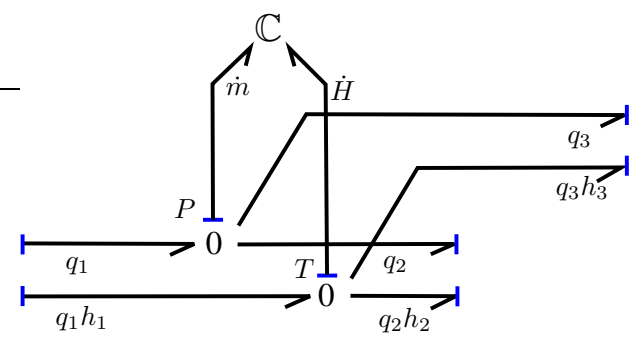

Figure 8. Causal bond graph of a junction with energy storage phenomena

from certain connected components. For instance an idealized version of a junction with no energy storage phenomenon is shown Figure 9. In that case one pair and only one pair of pressure and temperature variables will be imposed by an external connected component (here on port 1). It is not necessary that these effort variables be on the same inlet or outlet. The only constraint is that one pair and only one must be imposed from outside the junction component.

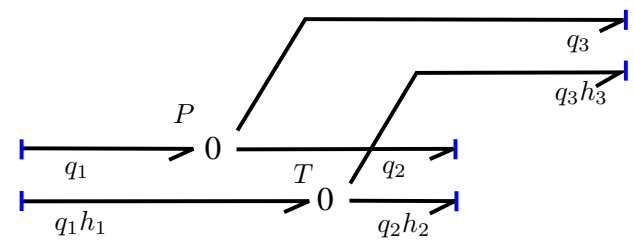

Figure 9. Causal bond graph of an idealized junction component

\section{F. Word bond graph}

The word bond graph for the whole system is built by connection of the different component bond graph representations identically to Figure 1 sketch. All connections are constituted of two ports for the fluid and the thermal domains respectively (Figure 7). The boundary conditions have been added by means of effort sources for the imposed pressures and temperatures. Since the leak has been neglected in the auxiliary equipment, the boundary condition BC6 only refers to the pressure. At this location and on the thermal bond, a flow source figures the source of heat. Finally the controlled temperature at outlet of the cooling systems is materialized in the bond graph by an effort detector placed on the thermal corresponding bond.

\section{CONClusion And Future Works}

This paper has presented the application of the physical structure analysis on each component of the intermediary cooling system systems. This model has been validated against the reference model "heat exchanger" [5]. The main results of analysis have been presented in the context of the bond graph representation. It intrinsically carries the physical and analysis concepts required for the physical structure analysis presented. The perspectives are to complete the studies at the global level for the whole system to ensure the causal consistency in the connection of the different component models and estimation of the state variables which are based on model inversion.

\section{REFERENCES}

[1] F. Lorenz, "Modelling System 1," users manual, Lorenz Simulation, Liege, Belgium, 1997.

[2] D.C. Karnopp, D.L. Margolis, and R.C. Rosenberg, "System Dynamics: Modeling, Simulation, and Control of Mechatronic Systems," Fifth Edition, John Wiley \& Sons, Inc. Hoboken, NJ, USA, 2012.

[3] The simulation program MS1, information available at: http://www.lorsim.be

[4] A. Jardin, W. Marquis-Favre, and D. Thomasset, "Bond Graph Sizing of Mechatronic Systems: Coupling of Inverse Modelling with Dynamic Optimization," In 6th Vienna International Conference on Methematical Modelling, Vienne, Austria, Feb, 11-13 2009, pp. 1929-1938.

[5] T. Bentaleb, M. T. Pham, D. Eberard, and W. Marquis-Favre, "Bond graph model of a water heat exchanger," in $30^{\text {th }}$ European Conference On Modelling And Simulation (ECMS), Regensburg, Germany, May $31^{\text {st }}$ - June $3^{\text {rd }} 2016$, pp. 333-339.

[6] Dymola 2017 FD01 Dynasim AB. Lund, Sweden, information available at: http://www.dymola.com

[7] D.C. Karnopp, D.L. Margolis, and R.C. Rosenberg, "System Dynamics: A Unified Approach," John Wiley \& Sons, Inc, 1990.

[8] F. M. White, "Fluid Mechanics," McGraw-Hill, seventh edition in si units edition, 2011.

[9] W. Wagner, and H-J. Kretzschmar, "International Steam Tables - Properties of Water and Steam based on the Industrial Formulation IAPWSIF97," Springer-Verlag Berlin Heidelberg, 2008. 